\title{
Far-Infrared Laser Spectroscopy of Phonon Difference Band Absorption in TICl
}

\author{
H. Lengfellner, R. Rindt and K.F. Renk \\ Institut für Angewandte Physik, Universität Regensburg, \\ Regensburg, Federal Republic of Germany \\ Received February 25, 1980
}

Far-infrared laser radiation is used to study the lattice absorption of a $\mathrm{TlCl}$ crystal over a large temperature range. We found a strong absorption band caused by phonon difference processes where longitudinal and transverse acoustic phonons with wave vectors at the Brillouin zone boundary are involved.

It is well known that most crystals which show Reststrahlen behavior are nearly transparent at low temperatures for far-infrared radiation at frequencies below the infrared active eigen-frequency. The crystals are nearly opaque at room temperature due to strong absorption by phonon combination processes [1]. We report experimental results which show that for $\mathrm{TlCl}$ a pronounced LA-TA two phonon difference band absorption occurs where transverse acoustic (TA) phonons and longitudinal acoustic (LA) phonons at the boundary of the Brillouin zone are involved.

For other crystals for which far-infrared difference band absorption has been studied no pronounced absorption by LA-TA phonon combination processes has been found. For alkali-halides it has been concluded from the temperature dependence of the absorption at fixed frequencies that absorption due to LA-TA combination processes is very weak [2]. Difference band absorption has been found in which mainly combinations of optical and acoustic phonons are involved. Similar results were reported for GaAs [3]. From the frequency dependence of the far-infrared absorption in InSb it has also been concluded that only weak LA-TA absorption occurs [4]. In this crystal the absorption by LA-TA processes is superimposed by absorption due to phonon sum processes.

We have measured the transmission of a $\mathrm{TlCl}$ crystal ( $2 \mathrm{~mm}$ thick) for radiation of a discharge pumped farinfrared laser. In order to obtain a survey of the transmission behavior of $\mathrm{TlCl}$ we have chosen laser radiation of frequencies at $0.89 \mathrm{THz}(337 \mu \mathrm{m}$ radiation of the HCN-laser), $1.36 \mathrm{THz}$ and $2.54 \mathrm{THz}$ (water vapor laser) and $1.75 \mathrm{THz}\left(\mathrm{D}_{2} \mathrm{O}\right.$ laser). These frequencies are below and above the infrared active eigen-frequency of $\mathrm{TlCl}$ at about $1.9 \mathrm{THz}[5,6]$. The radiation was detected with a Golay cell. In case of large sample transmission the intensity was reduced by absorption filters. For sample cooling we used a temperature variable optical cryostat with crystalline quartz windows. Due to the high power of the farinfrared radiation (10 to $100 \mathrm{~mW}$ ) it was possible to measure over a large transmission range without changing the sample.

The temperature dependence of the transmission for the different far-infrared laser lines is shown in Fig. 1. The transmission for radiation at $1.36 \mathrm{THz}$ and 0.89 $\mathrm{THz}$ decreases very strongly in the range of low temperature. The temperature dependence of the transmission at the higher frequencies is quite different. While at the lower temperatures the transmission changes slowly with temperature large changes are observed at temperatures above $100 \mathrm{~K}$.

Figure 2 gives a survey of the frequency dependence of the absorption coefficient obtained from an experiment in which we have determined the transmission corrigated for reflection losses [7]. It is clearly seen that a strongly temperature dependent absorption band arises at frequencies below the infrared active eigen-frequency with an absorption maximum at about $1 \mathrm{THz}$ and a cut-off at about $1.5 \mathrm{THz}$.

The far-infrared absorption of $\mathrm{TlCl}$ is caused by the anharmonicity of the crystal vibrations. The anhar- 


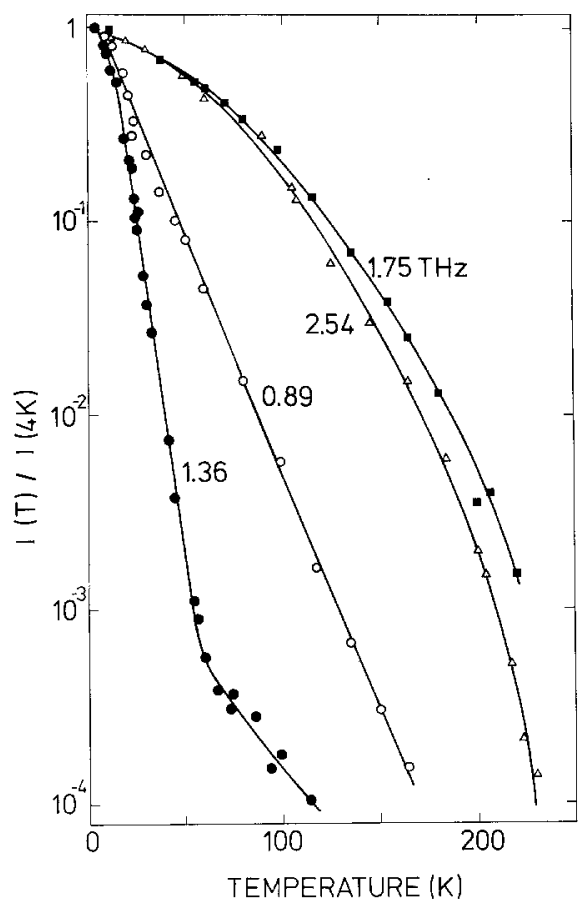

Fig. 1. Far-infrared transmission of a $\mathrm{TlCl}$ sample $(2 \mathrm{~mm}$ thickness). The values are normalised to the transmissions at $4 \mathrm{~K}$ which are $10^{-1}$ (for $0.89 \mathrm{THz}$ radiation), $5 \cdot 10^{-2}(1.36 \mathrm{THz}), 5 \cdot 10^{-3}(1.75$ $\mathrm{THz})$, and $5 \cdot 10^{-4}(2.54 \mathrm{THz})$, respectively

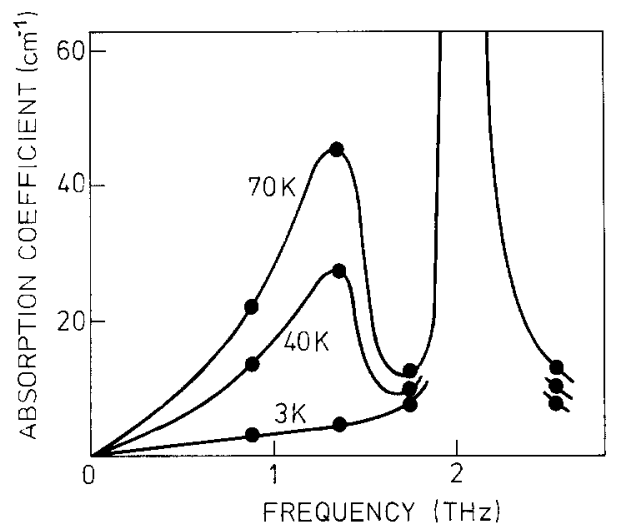

Fig. 2. Frequency dependence of the absorption coefficient estimated from the values at four frequencies (points)

monicity can give rise to absorption by phonon combination processes $[8,2]$. In the following we will show that the strongly temperature dependent absorption (Fig. 2) can be approximately described by a phonon difference process with the absorption coefficient

$\alpha\left(v_{L}, T\right)=A\left(v_{t}, v_{1}\right) \cdot\left[n\left(v_{t}, T\right)-n\left(v_{l}, T\right)\right]$

where $n\left(v_{t}, T\right)$ is the thermal occupation number of the phonon mode with frequency $v_{t}$ at the tempera- ture $T$ and $n\left(v_{l}, T\right)$ is the thermal occupation number of a phonon with frequency $v_{l}$. In a difference process a low frequency thermal phonon of frequency $v_{t}$ (TAphonon) and wave vector $q_{t}$ is destroyed and a high frequency phonon of frequency $v_{l}$ (LA-phonon) and wave vector $q_{l}$ is generated. Energy conservation requires that $v_{l}=v_{t}+v_{L}$ where $v_{L}$ is the frequency of the laser radiation.

Momentum conservation requires that the two phonons have the same wave vector $\left(q_{t}=q_{l}\right)$ because the wave vector of the far-infrared radiation is negligeable. The coefficient $A\left(v_{t}, v_{l}\right)$ which is a measure of the absorption strength at the frequency $v_{L}=v_{l}-v_{t}$ is determined by the combined density of states of the phonon difference processes modified by anharmonic coupling coefficients.

The temperature dependent proportion of the $a b-$ sorption coefficient at the laser frequencies $v_{L}=0.89$ $\mathrm{THz}$ and $1.36 \mathrm{THz}$ can well be described by (1) for low temperatures up to about $30 \mathrm{~K}$. This is shown in Fig. 3 where we have plotted $\ln \alpha$ versus $1 / T$. For very low temperatures where $n\left(v_{t}\right) \ll n\left(v_{t}\right) \ll 1$ the experimental absorption coefficient can be described by the low temperature approximation of (1) which is

$\alpha=A \cdot \exp \left(-h v_{t} / k T\right)$.

From the experimental slope $\ln \alpha$ versus $1 / T$ we have determined the frequency $v_{t}$, and the constant $A$ for the absorption at the two laser frequencies $v_{L}=0.89$

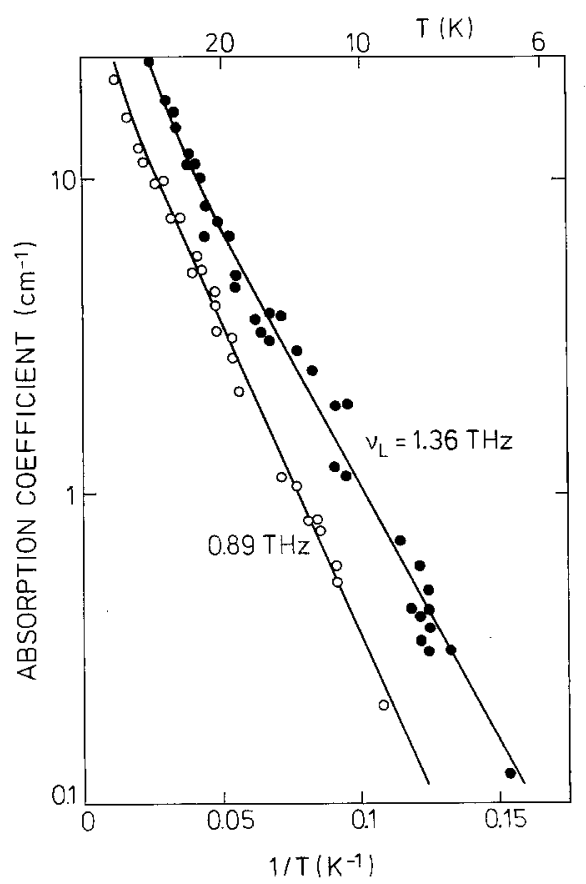

Fig. 3. Absorption coefficient at low temperatures. The experimental data can well be described by the solid lines calculated for absorption due to two-phonon difference processes according to (1) 
$\mathrm{THz}\left(A=35 \mathrm{~cm}^{-1}\right)$ and $1.36 \mathrm{THz}\left(A=40 \mathrm{~cm}^{-1}\right)$. We estimate that the experimental errors are about $5 \%$ for the values of $v_{t}$ and about $10 \%$ for $A$.

It is important to note that the temperature dependent absorption cannot be attributed to a two phonon sum process. In case of a sum process, two phonons of frequencies $v_{1}$ and $v_{2}$ (with $v_{1}+v_{2}=v_{L}$ ) are generated and the absorption can be described by $\alpha=A^{\prime}\left(n_{1}+n_{2}+1\right)$ where $n_{1}$ and $n_{2}$ are the thermal occupation numbers of the two phonons. The constant $A^{\prime}$ is the strength of the temperature dependent part of the sum process absorption coefficient as well as the absorption coefficient at very low temperature. From the discrepancy between the experimental value $\left(40 \mathrm{~cm}^{-1}\right)$ following from the temperature dependence of the absorption (at $1.36 \mathrm{THz}$ ) and the measured absolute absorption coefficient at $3 \mathrm{~K}$ where $\alpha=(5 \pm 2) \mathrm{cm}^{-1}$ (Fig. 2) we conclude that the temperature dependent absorption cannot be caused by a sum process. The same argument holds for the absorption of $0.89 \mathrm{THz}$ radiation where the low temperature value of the absorption coefficient is (3 $\pm 2) \mathrm{cm}^{-1}$ compared to $35 \mathrm{~cm}^{-1}$ which would follow from the temperature dependence. The origin of the (small) low temperature absorption is not clear, it could be due to defect-induced one-phonon absorption which is independent of temperature.

In order to show that the temperature dependent absorption is caused by two-phonon difference processes rather than processes in which three or more phonons are involved we have made an analysis of the high temperature transmission data. For twophonon difference processes one expects according to (1) at high temperatures $\left(k T>h v_{t}, h v_{l}\right)$ the dependence

$\alpha / T=A(k / h) v_{L} / v_{t} v_{l}$

where $k$ and $h$ are the Boltzmann and Planck constants, respectively. We find that for the absorption at $0.89 \mathrm{THz}$ the ratio $\alpha / T$ is independent of temperature (Fig. 4). This result demonstrates that the absorption is dominated by two-phonon processes.

If we calculate $\alpha / T$ from (1) with the low temperature values of $A, v_{t}$ and $v_{l}$, we obtain for temperatures above $30 \mathrm{~K}$ a small discrepancy between the predicted values and the experimental points (Fig. 4). The reason may be that the constant $A$ and the frequencies $v_{t}$ and $v_{l}$ change with increasing temperature. The high temperature values of $A$ and $v_{t}$ (and $v_{t}$ ) cannot be determined uniquely from the experiment because according to (3) the ratio $\alpha / T$ is related to the ratio $A / v_{t}\left(v_{t}+v_{L}\right)$. The dashed line in Fig. 4 is obtained for the results at $v_{L}=0.89 \mathrm{THz}$ with high temperature values $\left(v_{l}=1.0 \mathrm{THz}, \quad v_{l}=1.89 \mathrm{THz}, A\right.$

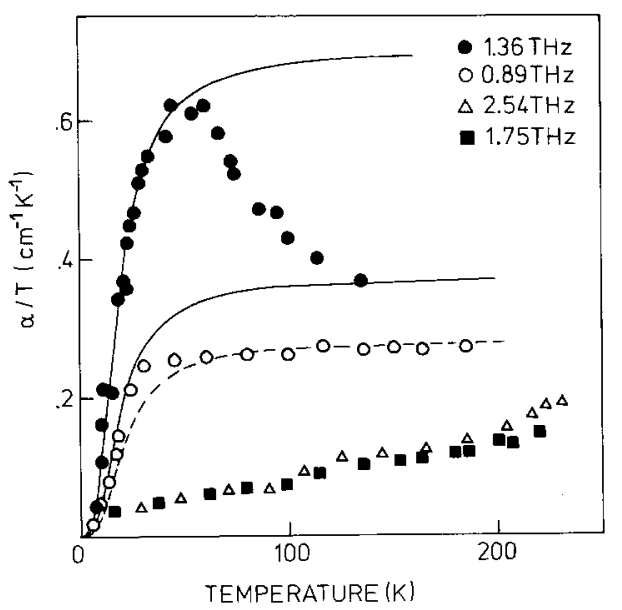

Fig. 4. The absorption at high temperatures (see text)

$=30 \mathrm{~cm}^{-1}$ ) which are only slightly different from the low temperaure values.

For the absorption at $1.36 \mathrm{THz}$ we find that $\alpha / T$ deviates strongly from the predicted curve for $T>50 \mathrm{~K}$ (Fig. 4). This gives evidence that the combined density of states of two-phonon difference processes has a cut-off very near $1.36 \mathrm{THz}$ and that this cut-off is changing to lower frequency with increasing temperature.

In Fig. 4 we have also plotted the ratio $\alpha / T$ determined for the radiation at $1.75 \mathrm{THz}$ and $2.54 \mathrm{THz}$. We find that $\alpha / T$ increases linearly with temperature. This is an indication that the absorption at these frequencies is caused mainly by three-phonon processes for which $\alpha / T$ increases proportionally to $T$ at high temperatures.

It is interesting that we can describe the absorption at $0.89 \mathrm{THz}$ and $1.36 \mathrm{THz}$ approximately by (1) rather than by a sum of various difference processes in which phonons at different frequencies $v_{t}$ and $v_{l}$ are involved. Therefore, our results give information on the phonon dispersion relation for $\mathrm{TlCl}$. The dispersion curves of $\mathrm{TlCl}$ which has $\mathrm{CsCl}$ structure are only known for phonons propagating along the (110) crystalline axis [9]. The frequencies of the low energy TA phonons propagating along the (110) direction have values less than $0.5 \mathrm{THz}$. Therefore, the TA phonons which we have found $(0.95 \mathrm{THz}$ and $0.76 \mathrm{THz}$ ) cannot be phonons propagating along the (110) direction. In order to find the assignment to phonons in the dispersion curve we used the similarity of the dispersion relation for the acoustic phonons in $\mathrm{TlCl}$ and $\mathrm{TlBr}$. The acoustic dispersion curves along the (110) direction are only slightly different for these two crystals. We assume that the curves are similar also for the other directions and use the dispersion relation of $\mathrm{TlBr}$ known from neutron experiments 
[10] as a guide for the dispersion relations of the acoustic phonons in TlCl. Then we can assign the combination band observed at $1.36 \mathrm{THz}$ to a difference process in which LA and TA phonons near the $\mathrm{X}$-point $(0.5,0,0)$ of the Brillouin zone are involved. The large strength of the absorption is indicating that the difference frequency changes only slowly in the surrounding of the $\mathrm{X}$-point. It follows from this assignment that the frequencies of the TA and LA phonons at the X-point $\left(v_{t}=0.76 \mathrm{THz}\right.$ and $v_{l}=2.12$ $\mathrm{THz}$ ) are slightly larger for $\mathrm{TlCl}$ than for $\mathrm{TlBr}$ where $v_{t}=0.7 \mathrm{THz}$ and $v_{l}=1.9 \mathrm{THz}[10]$. The combination band at $0.89 \mathrm{THz}$ seems to be due to processes in which LA and TA phonons at other points on the Brillouin zone boundary (near the $S$ and $Z$ points) are involved. According to our results, $1.36 \mathrm{THz}$ is about the highest frequency $v_{\max }$ of phonon difference combinations. A small decrease of $v_{\max }$ can result in a strong decrease of $\alpha / T$ as it is observed for temperatures above $50 \mathrm{~K}$ (Fig. 3).

It is known from the neutron experiments on $\mathrm{TlCl}$ [9] that the frequency of the lowest TA phonon branch at the (110) point is increasing strongly (by $20 \%$ ) between $4 \mathrm{~K}$ and $80 \mathrm{~K}$. Our experimental results (at $1.36 \mathrm{THz}$ and $0.89 \mathrm{THz}$ ) give evidence that frequencies of TA branches also at other points of the zone boundary increase with temperature.

For a more detailed understanding of the far-infrared absorption in $\mathrm{TlCl}$ it would be interesting to measure the absorption at further laser frequencies, which are available in optically pumped lasers. It would also be desirable to supplement our experiments by conventional far-infrared spectroscopy.

The method of laser spectroscopy should be useful to study other crystals which are strongly absorbing at far-infrared frequencies. We like to point out a fur- ther interesting application. We think that the absorption by difference processes can be used to detect very high frequency acoustic phonons. Since the absorption at the laser frequency is very sensitive to small temperature changes (Fig. 1) it should also be very sensitive to changes of the phonon occupation number occurring when non-thermal phonons are injected in a crystal. We think that infrared laser radiation can therefore be used for time dependent detection of high frequency phonons which have wave vectors at the zone boundary.

We thank W. Prettl for valuable discussions.

\section{References}

1. Hadni, A., Claudel, J., Gerbaux, X., Morlot, G., Munier, J.M.: Appl. Opt. 4, 487 (1965)

2. Stolen, R., Dransfeld, K.: Phys. Rev. 139, A 1295 (1965)

3. Stolen, R.: Phys. Rev. B 11, 767 (1975)

4. Koteles, E.S., Datars, W.R., Dolling, G.: Phys. Rev. B9, 572 (1974)

5. Jones, G.O., Martin, D.H., Mawer, P.A., Perry, C.H.: Proc. R. Soc. A 261, 10 (1961)

6. Lowndes, R.P., Martin, D.H.: Proc. R. Soc. A308, 473 (1969)

7. Lowndes, R.P.: Phys. Rev. B6, 1490 (1972)

8. Bilz, H., Genzel, L.: Z. Physik 169, 53 (1962)

9. Fujii, Y., Sakuma, T., Nakahava, J., Hoshino, S., Kobayashi, K., Fujii, A.: J. Phys. Soc. Jpn. 44, 1237 (1978)

10. Cowley, E.R., Okazaki, A.: Proc. R. Soc. A300, 45 (1967)

H. Lengfellner

R. Rindt

K.F. Renk

Institut für Angewandte Physik

Universität Regensburg

Universitätsstraße 31

D-8400 Regensburg

Federal Republic of Germany 